\title{
Characteristic of Enterococcus faecium clinical isolates with quinupristin/ dalfopristin resistance in China
}

\author{
Shanshan Wang ${ }^{1 \dagger}$, Yinjuan Guo ${ }^{1 \dagger}$, Jingnan $\mathrm{Lv}^{1}$, Xiuqin $\mathrm{Qi}^{1}$, Dan $\mathrm{Li}^{1}$, Zengqiang Chen ${ }^{1}$, Xueqing Zhang ${ }^{1}$, \\ Liangxing Wang ${ }^{2^{*}}$ and Fangyou $\mathrm{Yu}^{1^{*}}$
}

\begin{abstract}
Background: Quinupristin/dalfopristin (Q/D) is a valuable alternative antibiotic to vancomycin for the treatment of multi-drug resistant Enterococcus faecium infections. However, resistance to Q/D in E. faecium clinical isolates and nosocomial dissemination of Q/D-resistant E. faecium have been reported in several countries and should be of concern.

Results: From January 2012 to December 2015, 911 E. faecium clinical isolates were isolated from various specimens of inpatients at the first Affiliated Hospital of Wenzhou Medical University located in Wenzhou, east China. Of 911 E. faecium clinical isolates, 9 (1.0\%, 9/911) were resistant to Q/D, with the Q/D MIC values of $64 \mathrm{mg} / \mathrm{L}(1), 32 \mathrm{mg} / \mathrm{L}(1), 16 \mathrm{mg} / \mathrm{L}(3)$, $8 \mathrm{mg} / \mathrm{L}(1)$ and $4 \mathrm{mg} / \mathrm{L}(3)$ determined by broth microdilution. All Q/D-resistant isolates were susceptible to vancomycin, tigecycline and teicoplanin but resistant to penicillin, ampicillin and erythromycin. vatE was only found in one Q/D-resistant $E$. faecium isolate while vatD was not detected in any of the isolates tested. 8 of 9 Q/D-resistant $E$. faecium isolates were found be positive for both $\mathrm{erm} B$ and $\mathrm{msrC}$. The combinations of Q/D resistance determinants were ermB-msrC (7 isolates) and ermB-msrC-vatE (one isolate). ST78, ST761, ST94, ST21 and ST323 accounted for 4, 2, 1, 1 and 1 isolate, respectively, among which ST78 was the prevalent ST.
\end{abstract}

Conclusion: Q/D-resistant E. faecium clinical isolates were first described in China. Carriage of vatE, ermB and msrC was responsible for $\mathrm{Q} / \mathrm{D}$ resistance.

Keywords: Enterococcus faecium, Quinupristin/dalfopristin, Resistance

\section{Background}

Enterococci, which are the normal commensals in the intestinal tract of humans and animals, are the common cause of nosocomial infections. Enterococcus faecalis and Enterococcus faecium are responsible for most of enterococcal infections, such as urinary-tract infections, intra-abdominal infections, bacteremia, and endocarditis [1]. With an intrinsic and acquired resistance to some antimicrobial agents, Enterococci have become important nosocomial pathogens [2]. Infections caused by multidrug -resistant Enterococci, especially multiple resistances to vancomycin, penicillin, and aminoglycoside (high-level

\footnotetext{
*Correspondence: 38805@163.com; wzjxyfy@163.com

${ }^{\dagger}$ Equal contributors

2Department of Respiratory Medicine, the First Affiliated Hospital of Wenzhou Medical University, Wenzhou 325000, China

'Department of Laboratory Medicine, the First Affiliated Hospital of Wenzhou Medical University, Wenzhou 325000, China
}

resistance), are of a major concern, making enterococcal infections a serious and life-threatening disease [1]. Increase of vancomycin-resistant Enterococci (VRE) limits the selection of vancomycin for treatment of enterococcal infections. Therefore, attention has been directed toward the alternatives for the treatment of enterococcal infections, especially VRE infections.

Quinupristin/dalfopristin(Q/D) is a combination of streptogramins B (quinupristin) and streptogramins A (dalfopristin) at 30:70 ratio. Streptogramins A and B are bacteriostatic when used separately but act synergistically when combined. The synergic behavior of the combination results in $\mathrm{Q} / \mathrm{D}$ rapidly bactericidal against the majority of Gram-positive organisms [3]. Clinically, Q/D is mainly used for the treatment of infections caused by multidrug-resistant Gram-positive cocci $[4,5]$. Q/D is effective against $E$. faecium, but not against $E$. faecalis [6]. 
As E. faecalis isolates possess a chromosomal gene named lsa responsible for lincosamide and streptogramin A resistance, which results in all E. faecalis with intrinsic resistance to Q/D [2]. Q/D has been successfully used for the treatment of vancomycin-resistant $E$. faecium (VREf) infections $[7,8]$. The combination of $\mathrm{Q} / \mathrm{D}$ and high-dose ampicillin was used successfully for treating persistent bacteremia and endocarditis caused by VREf $[5,9]$. Q/D is a valuable alternative to vancomycin for the treatment of multi-drug resistant $E$. faecium infections. However, resistance to $\mathrm{Q} / \mathrm{D}$ in E. faecium clinical isolates and nosocomial dissemination of $\mathrm{Q} / \mathrm{D}$-resistant $E$. faecium have been found in several countries [10-13]. Although the prevalence of $\mathrm{Q} / \mathrm{D}$ resistance among these isolates from humans is still low, emergence and dissemination of Q/D-resistant E. faecium limit the therapeutic option of successful treatment of VREf infections. However, Q/D resistance among E. faecium isolates from inpatients has not been reported in China. The aim of the present study was to elucidate the prevalence of Q/D resistance among E. faecium isolates from the various specimens of inpatients at a tertiary teaching hospital between January 2012 and December 2015.

\section{Methods}

Collection of clinical isolates and $E$. faecium identification From January 2012 to December 2015, a total of 911 non-duplicate E. faecium isolates (single isolate per patient) from various specimens of inpatients in the first Affiliated Hospital of Wenzhou Medical University located in Wenzhou, east China, were collected consecutively for investigating the prevalence of Q/D resistance. When multiple E. faecium isolates were isolated from the same patient, the first isolated strain was included. The included isolates were identified as E. faecium using Gram's stain, catalase test and Vitek-2 microbiology analyzer (bioMe'rieu, Marcy l'Etoile, France). Q/D-resistant isolates were re-identified as E. faecium using MatrixAssisted Laser Desorption/ Ionization Time of Flight Mass Spectrometry (MALDI-TOF-MS) (bioMe'rieux, Marcy l'Etoile, France) and PCR amplifying 16S rRNA gene. S. aureus ATCC25923, Escherichia coli ATCC25922 and Pseudomonas aeruginosa ATCC27853 were used as control strains for identification of bacteria. The Ethics Committee of the first Affiliated Hospital of Wenzhou Medical University exempted this study from review because the present study focused on bacteria.

\section{Antimicrobial susceptibility testing}

The antimicrobial susceptibility testing for E. faecium clinical isolates was determined by Vitek-2 microbiology analyzer (bioMe'rieu, Marcy l'Etoile, France) in accordance with the manufactory's instructions. Q/D resistance initially determined by Vitek-2 microbiology analyzer was reconfirmed using disk diffusion method were according to the guidelines provided by the Clinical and Laboratory Standards Institute (CLSI) [14]. Q/D minimum inhibitory concentration (MIC) values for $\mathrm{Q} / \mathrm{D}$ resistant $E$. faecium clinical isolates were determined twice using broth microdilution method operated by two separate operators in accordance with the guidelines recommended by CLSI [14]. Q/D (Synercid, Astellas Pharma, Inc.) was kindly provided by Pro. Nobumichi Kobayashi from Sapporo Medical University (Japan). S. aureus ATCC25923, E. coli ATCC25922 and P. aeruginosa ATCC27853 were used as control strains for antimicrobial susceptibility testing.

\section{Detection of determinants responsible for $\mathrm{Q} / \mathrm{D}$ resistance}

The genes responsible for resistance to streptogramin $\mathrm{B}$ antibiotics including erm (ermA ermB and ermC) and $m s r C$, and streptogramin A antibiotics including vat (vatD and vatE) were detected by PCR assays with specific primers and reaction conditions described previously [15-17]. DNA sequencing was used for the identification of genotype of the genes tested.

\section{Multi-locus sequence typing (MLST)}

MLST of E. faecium isolates was performed using amplification of internal fragments of the seven housekeeping genes including $a d k, a t p A, d d l, g y d, g d h, p u r K$ and $p s t S$ of this organism as described previously [18]. Following purification and sequencing of these genes, the sequences were compared with the existing sequences available on the MLST website for E. faecium (http://efaecium.mlst.net), and STs were determined according to the allelic profiles.

\section{Results and discussion}

\section{Prevalence of $Q / D$ resistance among $E$. faecium clinical isolates}

Among 911 E. faecium clinical isolates over the study period, 9 (1.0\%, 9/911) were resistant to Q/D determined by the Vitek-2 Automated Microbiology Analyzer with GPS card and disk diffusion method. The Q/D MIC values for 9 Q/D-resistant E. faecium clinical isolates determined by broth microdilution method were as follows: $64 \mathrm{mg} / \mathrm{L}$, $1 ; 32 \mathrm{mg} / \mathrm{L}, 1 ; 16 \mathrm{mg} / \mathrm{L}, 3 ; 8 \mathrm{mg} / \mathrm{L}, 1$ and $4 \mathrm{mg} / \mathrm{L}, 3$ (Table 1). All Q/D-resistant isolates were susceptible to vancomycin, tigecycline and teicoplanin but resistant to penicillin, ampicillin and erythromycin. Eight (88.9\%) of 9 isolates with $\mathrm{Q} / \mathrm{D}$ resistance were resistant to moxiofloxacin, levofloxacin and ciprofloxacin. Five, 1 and 5 isolates were resistant to gentamicin (high-level resistance), linezolid and nitrofurantoin, respectively (Table 1 ). As Q/D is not available in China, the patients infected by these Q/D-resistant E. faecium isolates were not subject to be treated by $\mathrm{Q} / \mathrm{D}$. Therefore, we speculate that the acquisition of $\mathrm{Q} / \mathrm{D}$ resistance among $E$. faecium clinical 
Table 1 The characteristics of Q/D-resistant E. faecium isolates

\begin{tabular}{|c|c|c|c|c|c|c|}
\hline No. & Specimen & ST & $\begin{array}{l}\text { Q/D MIC value } \\
(\mathrm{mg} / \mathrm{l})\end{array}$ & Antimicrobial resistance profile ${ }^{a}$ & Antimicrobial susceptibility profile ${ }^{a}$ & $\begin{array}{l}\text { Q/D resistance } \\
\text { determinants }\end{array}$ \\
\hline 1 & pus & 78 & 16 & MOX, GEN(H), P, E, LZD, F, LEV, AMP, CIP & TEC, TGC, TET, VAN & ermB, msrC \\
\hline 2 & urine & 78 & 32 & MOX, GEN(H), P, E, LEV, AMP, CIP & LZD, TEC, F, TGC, TET, VAN & ermB, msrC \\
\hline 3 & catheter & 94 & 16 & GEN(H), P, E, LEV, AMP, CIP, & MOX, LZD, TEC, F, TGC, TET, VAN & \\
\hline 4 & exudate & 761 & 8 & $P, E, A M P$ & LEV, CIP, MOX, GEN(H), TEC, F, TGC, TET, VAN, LZD & ermB, msrC \\
\hline 5 & wound & 21 & 64 & MOX, GEN(H), P, E, F, LEV, AMP, CIP & LZD, TEC, TGC, TET, VAN & ermB, msrC, vate \\
\hline 6 & exudate & 323 & 4 & MOX, P, E, F, LEV, AMP, CIP & GEN(H), LZD, TEC, TGC, TET, VAN & ermB, msrC \\
\hline 7 & urine & 78 & 4 & MOX, P, E, LEV, AMP, CIP & GEN(H), LZD, F, TEC, TGC, TET, VAN & ermB, msrC \\
\hline 8 & blood & 761 & 16 & MOX, P, E, LEV, AMP, CIP, TET, F & GEN(H), LZD, TEC, TGC, VAN & ermB, msrC \\
\hline 9 & tissue & 78 & 4 & MOX, GEN(H), P, E, LEV, AMP, CIP, F & LZD, TEC, TGC, TET, VAN & ermB, msrC \\
\hline
\end{tabular}

${ }^{a} P$ penicillin, AMP ampicillin, E erythromycin, TET tetracycline, TGC tigecycline. GEN(H) high-level gentamicin, CIP ciprofloxacin, MOX moxifloxacin, $L E V$ levofloxacin, $F$ nitrofurantoin, LZD linezolid, VAN vancomycin, TEC teicoplanin

isolates in the present study was not associated with the consumption of $Q / D$. The previous study reported that the extensive in-feed use of virginiamycin showed full cross-resistance with Q/D selected for streptograminresistant enterococci and resulted in a reservoir of resistance genes in production animals [19]. Q/D resistance determinants from animal-associated enterococcal isolates can spread to E. faecium human isolates [20]. However, in the present study, it remains unknown whether these Q/D-resistant E. faecium clinical isolates was indeed associated with the isolates from animals. To our knowledge, the present study is the first report of $\mathrm{Q} / \mathrm{D}$ resistance among $E$. faecium clinical isolates in China. Up to now, although the prevalence of Q/D resistance among E. faecium clinical isolates was very low, the intermediate resistance to $\mathrm{Q} / \mathrm{D}$ was relatively high. A investigation from Japan reported that none was resistant to Q/D while 28 (17.6\%, 28/159) were intermediate resistant to this antimicrobial agent $(\mathrm{MIC}=2 \mathrm{mg} / \mathrm{L}$ ) among 159 E. faecium isolates from clinical specimens in a Japanese hospital from 1997 to 2006 [21]. A study from Greek reported that 250 of 865 (28.9 \%) E. faecium isolates from patients of eight Greek hospitals between 2005 and2006 were intermediate-resistant to Q/D $(\mathrm{MICs}=1.5-$ $4 \mathrm{mg} / \mathrm{L}$ ) [22]. In another report, all 60 primary clinical isolates of E. faecium with resistance to glycopeptides were fully susceptible to Q/D, with MIC50 and MIC90 values of $1.0 \mathrm{mg} / \mathrm{L}$ and $1.5 \mathrm{mg} / \mathrm{L}$ [23]. These previous studies and our study support the evidence that $\mathrm{Q} / \mathrm{D}$ is still an effective and valuable antimicrobial agent for treating infections caused by multi-resistant $E$. faecium, even VREf. Nevertheless, the emergence of $\mathrm{Q} / \mathrm{D}$ resistance, especially increased intermediate resistance to this antimicrobial agent, has become a concern. Moreover, a high prevalence $(10.0 \%)$ of Q/D resistance among E. faecium clinical isolates was found in Korea, which was associated with both clonal spread and the sporadic emergence of Q/D-resistant isolates [12]. Hsueh et al. also reported a similar high prevalence (9\%) of Q/D resistance among vancomycinresistant E. faefium clinical isolates in Taiwan [24]. As our study did not investigate the prevalence of intermediate resistance to Q/D among E. faecium clinical isolates, it remains unclear whether there was a trend of increase in intermediate resistance to $Q / D$, which should be further investigated.

\section{Determinants of $Q / D$ resistance among E. faecium clinical isolates}

The resistance to $Q / D$ is associated with enzymatic modification of the antibiotic, active transport or efflux mediated by an ATP-binding protein, and alteration of the target site [6]. Both resistance to streptogramin A and streptogramin $B$ is necessary for occurrence of $Q / D$ resistance [25]. Carriage of more than one streptogramin A resistance gene (vat or vga) is necessary for the occurrence of Q/D resistance in an organism [6]. The streptogramin A resistance genes found in $E$. faeciumare were vatD and vatE encoding acetyltransferases [6]. Modification of dalfopristin by the acetyltransferases VatD and VatE renders it ineffective, abolishing the synergy with quinupristin. vatD was initially found in an E. faecium isolate from a hospitalized patient in Europe [26].vatD and vatE have been found in E. faecium animal and human isolates in Europe, USA and Korea [6, 27, 28]. In contrast, some studies reported that none of the genes involved in the expression of dalfopristin resistance (vatD, vatE, vgaA and $v g a B$ ) were found in any Q/D-intermediate-resistant or -resistant E. faecium isolates [21, 22, 27]. Similarly, in the present study, vatE was only found in one Q/Dresistant $E$. faecium isolate while vatD was not detected in any of the isolates tested (Table 1). The most commonly known resistance to streptogramin $B$ in enterococci is the macrolides, lincosamides, and streptogramins B ( MLSB) resistance conferred by the erm genes (ermA, ermB and erm $C$ ) encoding an enzyme that dimethylates an adenine residue in the $23 \mathrm{~S}$ rRNA, which results in decreased 
binding of these antimicrobial agents [6]. In the present study, ermB was found among 8 of 9 Q/D-resistant $E$. faecium isolates. $v g b A$ encoding a streptogramininactivating enzyme (lyase) has been found rarely in the isolates of $E$. faecium, but $m s r C$ conferring resistance to streptogramin B antibiotics by active transport is commonly found in E. faecium isolates [29]. In the present study, 8 of 9 Q/D-resistant E. faecium isolates were found be positive for $m s r C$. The remaining one isolate was negative for all resistance genes tested. Currently, there has not been reported the combination of multiple streptogramin A resistance genes in E. faecium. However, combinations of the vatD-vgbA and ermB-vatD or vatE genes were found in $\mathrm{Q} / \mathrm{D}$-resistant isolates [10, 30, 31]. The vatE was more common than vatD in animal sources [11, 28, 32]. In the present study, the combinations of $\mathrm{Q} / \mathrm{D}$ resistance determinants were ermB-msrC (7 isolates) and ermB$m s r C$-vatE (one isolate).

\section{Molecular characteristics of Q/D-resistantE. Faecium clinical isolates}

The lineage clonal complex 17 (CC17) (ST17, ST18, and ST78) significantly associated with hospital infections emerges as the high-risk clone responsible for the worldwide spread of VERf [33]. A report from Poland showed the domination of representatives of lineages ST78 and ST17/18 (52.7 and 46.4\%, respectively) among consecutive E. faecium clinical isolates collected in 30 hospitals between May 2010 and June 2011 through prospective surveillance in Poland [34]. VERf isolates from 10 infected and 40 colonized inpatients from a single hospital in the north of Spain were assigned to ST17 by MLST [35]. In China, ST78 was the predominant MLST type among VERf clinical isolates [36]. However, the molecular characteristic of Q/D-resistant E. faecium clinical isolates is limited. Among $25 \mathrm{Q} / \mathrm{D}$-resistant E. faecium isolates from Korea, 10, 9 and 4 belonged to ST78, ST192 and ST203, respectively, with ST78 being the prevalent ST [12]. In the present study, among $9 \mathrm{Q} / \mathrm{D}$-resistant isolates, ST78, 761, 94, 21 and 323 accounted for 4, 2, 1, 1 and 1 isolate, respectively, with ST78 being the prevalent ST (Table 1). In another report, 5 STs including four STs of $\mathrm{CC} 17$ were identified in Q/D-intermediate resistant $E$. faecium clinical isolates [21]. In addition, only a VERf isolate from China was found to belong to ST323 [37]. The present study is the second report of an E. faecium ST323 isolate with $\mathrm{Q} / \mathrm{D}$ resistance associated with bloodstream infection.

\section{Conclusion}

In the present study, Q/D-resistant $E$. faecium clinical isolates were first described in China. We also identified that carriage of vatE, ermB and $m s r C$ was responsible for Q/D resistance in E. faecium clinical isolates.

\section{Abbreviations}

CLSI: Clinical and Laboratory Standards Institute; MALDI-TOF-MS: Matrix-Assisted Laser Desorption/ Ionization Time of Flight Mass Spectrometry; MIC: Minimum inhibitory concentration; MLST: Multi-locus sequence typing; Q/D: Quinupristin/ dalfopristin; VREf: Vancomycin-resistant Enterococcus faecium

\section{Acknowledgment}

We thank Pro. Nobumichi Kobayashi from Sapporo Medical University (Japan) for providing kindly Q/D for determining Q/D MICs.

\section{Funding}

The Program for Zhejiang Provincial Leading Team for S\&T innovation (2011R50018-11) and Zhejiang Provincial Program for the Cultivation of High-level Innovative Health talents to Dr. Fangyou Yu.

Availability of data and materials

Data and materials have been provided in the main manuscript.

\section{Authors' contributions}

SSW, YJG, JNL, XQQ and DL performed the laboratory measurements. FYY and LXW made substantial contributions to conception and design. ZQC, LXW and FYY revised the manuscript critically for important intellectual content. ZQC and XQZ participated in experimental design and data analysis. FYY drafted the manuscript. All authors read and approved the final manuscript.

\section{Competing interests}

The authors declare that they have no competing interests.

Consent for publication

Not applicable.

\section{Ethics approval and consent to participate}

The Ethics Committee of the first Affiliated Hospital of Wenzhou Medical University exempted this study from review because the present study focused on bacteria. Verbal informed consents were obtained from all participants.

Received: 17 July 2016 Accepted: 11 October 2016

Published online: 21 October 2016

\section{References}

1. Arias CA, Murray BE. The rise of the Enterococcus: beyond vancomycin resistance. Nat Rev Microbiol. 2012;10(4):266-78.

2. Miller WR, Munita JM, Arias CA. Mechanisms of antibiotic resistance in enterococci. Expert Rev Anti-Infect Ther. 2014;12(10):1221-36.

3. Jones RN, Ballow CH, Biedenbach DJ, Deinhart JA, Schentag JJ. Antimicrobial activity of quinupristin-dalfopristin (RP 59500, Synercid) tested against over 28,000 recent clinical isolates from 200 medical centers in the United States and Canada. Diagn Microbiol Infect Dis. 1998;31(3):437-51.

4. Lentino JR, Narita M, Yu VL. New antimicrobial agents as therapy for resistant gram-positive cocci. Eur J Clin Microbiol Infect Dis. 2008;27(1):3-15.

5. Bethea JA, Walko CM, Targos PA. Treatment of vancomycin-resistant enterococcus with quinupristin/dalfopristin and high-dose ampicillin. Ann Pharmacother. 2004;38(6):989-91.

6. Hershberger E, Donabedian S, Konstantinou K, Zervos MJ. Quinupristindalfopristin resistance in gram-positive bacteria: mechanism of resistance and epidemiology. Clin Infect Dis. 2004;38(1):92-8.

7. Tan TY, Pitman I, Penrose-Stevens A, Simpson BA, Flanagan PG. Treatment of a vancomycin-resistant Enterococcus faecium ventricular drain infection with quinupristin/dalfopristin and review of the literature. J Infect. 2000:41(1):95-7.

8. Linden PK, Moellering Jr RC, Wood CA, Rehm SJ, Flaherty J, Bompart F, Talbot GH, Synercid Emergency-Use Study G. Treatment of vancomycinresistant Enterococcus faecium infections with quinupristin/dalfopristin. Clin Infect Dis. 2001;33(11):1816-23.

9. Thompson RL, Lavin B, Talbot GH. Endocarditis due to vancomycin-resistant Enterococcus faecium in an immunocompromised patient: cure by administering combination therapy with quinupristin/dalfopristin and high-dose ampicillin. South Med J. 2003;96(8):818-20.

10. De Graef EM, Decostere A, De Leener E, Goossens H, Baele M, Haesebrouck F. Prevalence and mechanism of resistance against macrolides, lincosamides, and streptogramins among Enterococcus faecium isolates from food-producing animals and hospital patients in Belgium. Microb Drug Resist. 2007;13(2):135-41. 
11. Donabedian SM, Perri MB, Vager D, Hershberger E, Malani P, Simjee S, Chow J, Vergis EN, Muder RR, Gay K, et al. Quinupristin-dalfopristin resistance in Enterococcus faecium isolates from humans, farm animals, and grocery store meat in the United States. J Clin Microbiol. 2006:44(9):3361-5.

12. Oh WS, Ko KS, Song JH, Lee MY, Park S, Peck KR, Lee NY, Kim CK, Lee H, Kim SW, et al. High rate of resistance to quinupristin-dalfopristin in Enterococcus faecium clinical isolates from Korea. Antimicrob Agents Chemother. 2005;49(12):5176-8.

13. Werner G, Klare I, Spencker FB, Witte W. Intra-hospital dissemination of quinupristin/dalfopristin- and vancomycin-resistant Enterococcus faecium in a paediatric ward of a German hospital. J Antimicrob Chemother. 2003;52(1):113-5.

14. CLSI. Performance standards for antimicrobial susceptibility testing, 24th informational supplement (M100-S24). Wayne: Clinical and Laboratory Standards Institute; 2014

15. Soltani M, Beighton D, Philpott-Howard J, Woodford N. Mechanisms of resistance to quinupristin-dalfopristin among isolates of Enterococcus faecium from animals, raw meat, and hospital patients in Western Europe. Antimicrob Agents Chemother. 2000;44(2):433-6.

16. McDermott PF, Cullen P, Hubert SK, McDermott SD, Bartholomew M, Simjee S, Wagner DD. Changes in antimicrobial susceptibility of native Enterococcus faecium in chickens fed virginiamycin. Appl Environ Microbiol. 2005;71(9):4986-91.

17. Sutcliffe J, Grebe T, Tait-Kamradt A, Wondrack L. Detection of erythromycinresistant determinants by PCR. Antimicrob Agents Chemother. 1996;40(11):2562-6.

18. Homan WL, Tribe D, Poznanski S, Li M, Hogg G, Spalburg E, Van Embden JD, Willems RJ. Multilocus sequence typing scheme for Enterococcus faecium. J Clin Microbiol. 2002:40(6):1963-71.

19. Jensen LB, Hammerum AM, Bager F, Aarestrup FM. Streptogramin resistance among Enterococcus faecium isolated from production animals in Denmark in 1997. Microb Drug Resist. 2002:8(4):369-74.

20. Simjee S, White DG, Meng J, Wagner DD, Qaiyumi S, Zhao S, Hayes JR, McDermott PF. Prevalence of streptogramin resistance genes among Enterococcus isolates recovered from retail meats in the Greater Washington DC area. J Antimicrob Chemother. 2002;50(6):877-82.

21. Isogai N, Urushibara N, Kawaguchiya M, Ghosh S, Suzaki K, Watanabe N, Quinones D, Kobayashi N. Characterization of Enterococcus faecium with macrolide resistance and reduced susceptibility to quinupristin/dalfopristin in a Japanese hospital: detection of extensive diversity in erm(B)-regulator regions. Microb Drug Resist. 2013;19(4):298-307.

22. Karanika M, Prati A, Kiritsi M, Spiliopoulou I, Neonakis I, Anifantaki M, Petinaki E. Reduced susceptibility to quinupristin/dalfopristin in Enterococcus faecium in Greece without prior exposure to the agent. Int J Antimicrob Agents. 2008;31(1):55-7.

23. Abb J. In vitro activities of tigecycline, daptomycin, linezolid and quinupristin/dalfopristin against glycopeptide-resistant Enterococcus faecium. Int J Antimicrob Agents. 2007;29(3):358-60.

24. Hsueh PR, Chen WH, Teng LJ, Luh KT. Nosocomial infections due to methicillin-resistant Staphylococcus aureus and vancomycin-resistant enterococci at a university hospital in Taiwan from 1991 to 2003: resistance trends, antibiotic usage and in vitro activities of newer antimicrobial agents. Int J Antimicrob Agents. 2005;26(1):43-9.

25. Werner G, Klare I, Witte W. Molecular analysis of streptogramin resistance in enterococci. Int J Med Microbiol. 2002;292(2):81-94.

26. Rende-Fournier R, Leclerca R, Galimand M, Duval J, Courvalin P. Identification of the sat $A$ gene encoding a streptogramin $A$ acetyltransferase in Enterococcus faecium BM4145. Antimicrob Agents Chemother. 1993;37(10):2119-25.

27. Hwang IY, Ku HO, Lim SK, Lee KJ, Park CK, Jung GS, Jung SC, Park YH, Nam HM. Distribution of streptogramin resistance genes and genetic relatedness among quinupristin/dalfopristin-resistant Enterococcus faecium recovered from pigs and chickens in Korea. Res Vet Sci. 2010;89(1):1-4.

28. Jackson CR, Fedorka-Cray PJ, Barrett JB, Hiott LM, Woodley TA. Prevalence of streptogramin resistance in enterococci from animals: identification of vatD from animal sources in the USA. Int J Antimicrob Agents. 2007;30(1):60-6.

29. Portillo A, Ruiz-Larrea F, Zarazaga M, Alonso A, Martinez JL, Torres C. Macrolide resistance genes in Enterococcus spp. Antimicrob Agents Chemother. 2000;44(4):967-71.

30. Hammerum AM, Flannagan SE, Clewell DB, Jensen LB. Indication of transposition of a mobile DNA element containing the vat(D) and erm(B) genes in Enterococcus faecium. Antimicrob Agents Chemother. 2001;45(11):3223-5.
31. Jensen $L B$, Hammerum $A M$, Aarestrup FM. Linkage of vat(E) and erm(B) in streptogamin-resistant Enterococcus faecium isolates from Europe. Antimicrob Agents Chemother. 2000;44(8):2231-2.

32. Fard RM, Heuzenroeder MW, Barton MD. Antimicrobial and heavy metal resistance in commensal enterococci isolated from pigs. Vet Microbiol. 2011;148(2-4):276-82.

33. Leavis HL, Bonten MJ, Willems RJ. Identification of high-risk enterococcal clonal complexes: global dispersion and antibiotic resistance. Curr Opin Microbiol. 2006;9(5):454-60.

34. Gawryszewska I, Zabicka D, Bojarska K, Malinowska K, Hryniewicz W, Sadowy E. Invasive enterococcal infections in Poland: the current epidemiological situation. Eur J Clin Microbiol Infect Dis. 2016;35(5):847-56.

35. Valdezate S, Labayru C, Navarro A, Mantecon MA, Ortega M, Coque TM, Garcia M, Saez-Nieto JA. Large clonal outbreak of multidrug-resistant CC17 ST17 Enterococcus faecium containing Tn5382 in a Spanish hospital. J Antimicrob Chemother. 2009;63(1):17-20.

36. Yang J, Jiang Y, Guo L, Ye L, Ma Y, Luo Y. Prevalence of diverse clones of vancomycin-resistant Enterococcus faecium ST78 in a Chinese hospital. Microb Drug Resist. 2016;22(4):294-300.

37. Zheng B, Tomita H, Xiao YH, Wang S, Li Y, Ike Y. Molecular characterization of vancomycin-resistant enterococcus faecium isolates from mainland China. J Clin Microbiol. 2007:45(9):2813-8.

\section{Submit your next manuscript to BioMed Central and we will help you at every step:}

- We accept pre-submission inquiries

- Our selector tool helps you to find the most relevant journal

- We provide round the clock customer support

- Convenient online submission

- Thorough peer review

- Inclusion in PubMed and all major indexing services

- Maximum visibility for your research

Submit your manuscript at www.biomedcentral.com/submit
) Biomed Central 